\title{
EDITORIAL
}

\section{Restoration of accommodation: new perspectives}

\section{Restauração da acomodação: novas perspectivas}

Tracy Schroeder Swartz ${ }^{1}$, Karolinne Maia Rocha ${ }^{2}$, Mitch Jackson ${ }^{3}$, David HK MA , Daniel Goldberg ${ }^{5}$, AnnMarie Hipsley

Presbyopia is the loss of accommodative ability that occurs with age. Current accommodative theory postulates that the lens is primarily responsible for the refractive change that allows us to read. Our understanding of this process has grown substantially with the advent of new technologies, including ultrasound biomicroscopy (UBM), endoscopy, optical coherence tomography $(\mathrm{OCT})$, ray-tracing and wavefront analysis. Goldberg's Postulate incorporates all elements of the zonular apparatus into the phenomenon of accommodation ${ }^{(1)}$. The ciliary body contracts during accommodation. Biometry has shown that the lens thickness increases and the anterior chamber depth decreases ${ }^{(2)}$. It has also demonstrated the lens capsule steepens, as the posterior-lens surface moves backwards ${ }^{(2)}$

In addition, there is a decrease in the distance from scleral spur to the ora serrata. UBM identified an attachment zone of the posterior zonules adjacent to the ora, and contraction of these zonules is thought to be the etiology of the decrease in distance found with accommodation. This complex action of the zonules is suspected to be reciprocal. As the same time the anterior zonules relax, reducing their tension on the lens such that the lens changes shape anteriorly, the posterior zonules contract, moving the posterior capsule backward. This vitreal-zonular complex stiffens with age, losing its elasticity ${ }^{(1-3)}$. The age-related changes in these structures and their biomechanical interactions with the ciliary-lens complex may contribute to presbyopia ${ }^{(3)}$. It has been newly discovered that there are also changes in extralenticular structures which may have an impact on the loss of accommodation which were previously deemed to be of very little importance, namely the sclera and choroid ${ }^{(2)}$.

All ocular tissues are made of collagen and are impacted like all other connective tissues by age. Ocular rigidity has been correlated with age ${ }^{(4)}$ and the sclera undergoes scleral sclerosis as well as metabolic physiological stress. With the loss of elasticity, the more rigid sclera elicits compression and loading stresses upon underlying structures, specifically those related to accommodative function. Increased ocular rigidity affects other tissues as well, including ocular blood flow through the sclera and optic nerve. It has been correlated to the pathogenesis of macular degeneration ${ }^{(5)}$ and other age-related eye diseases ${ }^{(6)}$. Ocular rigidity may not only impact the loss of visual accommodation but also have more extensive clinical significance.

The impact of age on the lenticular-based model of loss of accommodation is well documented. The amount of accommodation lost with age related to extralenticular apparatus (primarily the zonules, choroid, and sclera) was only recently investigated ${ }^{(7)}$. It is also now known that the sclera becomes less deformable during accommodation in the nasal area with age ${ }^{(2)}$. New models suggest up to 2 diopters that might be contributed by extralenticular structures ${ }^{(7)}$.

To date, there has been at least up to 1-2 diopters of a loss of accommodation unaccounted for that might be contributed by extralenticular structures ${ }^{(7)}$. The investigation improving ocular resilience for the restoration of accommodation is of clinical importance. The Laser anterior ciliary excision procedure is designed to do so by altering biomechanical scleral properties. This is achieved by creating micropores in a matrix over four oblique quadrants. The VisioLite erbium-YAG laser creates micro-excisions in critical zones of physiologic importance overlying the ciliary-lens complex. The matrix pattern of nine $600 \mu \mathrm{m}$ laser spots in the sclera of each quadrant aims to increase plasticity in those regions across the anterior globe. The Laser anterior cilary excision primary mechanism of action is to decrease scleral resistive forces in order to restore accommodative ability in the aging eye by increasing resultant ciliary muscle constriction (Figure 1).

Laboratory studies of ocular rigidity demonstrated use of a reference model, and effect of laser anterior ciliary ablation on aging porcine eyes in vitro ${ }^{(8)}$. Investigators concluded that the scleral crosslinking method might be useful for correlation of age-related rigidity, as well as the efficacy of the laser anterior ciliary ablation procedure to decrease ocular rigidity ${ }^{(8)}$.

Submitted for publication: February 12, 2014

Accepted for publication: February 13, 2014

${ }_{1}^{1}$ Madison, Alabama, USA

${ }^{2}$ Cleveland Clinic Foundation, Cole Eye Institute, Ohio, USA.

3 Jackson Eye, Chicago, Illinois, USA.

${ }^{4}$ Chang Gung Memorial Hospital, Taipei, Taiwan.

${ }^{5}$ Drexel College of Medicine, Philadelphia, PA.; Atlantic Eye Physicians, Little Silver NJ, USA.

${ }^{6}$ Ace Vision Group, Newark California, USA.
Funding: No specific financial support was available for this study

Disclosure of potential conflicts of interest: T.S. Swartz, None; K.M. Rocha, None; M. Jackson, None; D.H.K. Ma, None; D. Goldberg, None; A. Hipsley, None.

Correspondence address: Karolinne Rocha. Cleveland Clinic Foundation. Cole Eye Institute, Ohio, USA - E-mail: karolinnemaia@gmail.com; ahipsley@acevisiongroup.com 
Several studies using laser anterior ciliary ablation have been performed to demonstrate its use in presbyopia. A study of 134 eyes of 67 patients who underwent bilateral procedure showed that some accommodative ability was restored. Accommodation was measured objectively using wavefront analysis. Minimum accommodative amplitude was 1.50D, and maximum was $5.50 \mathrm{D}$, and a mean of $3.08 \mathrm{D}$ of accommodative ability was achieved $(\mathrm{SD}=0.07 ; \mathrm{p}=0.032)^{(9)}$.

Fifty-two eyes of 26 presbyopic patients were enrolled in an IRB registered pilot study at the Chang Gung Memorial Hospital, Taipei Taiwan. Patients older than 40 years old with a demonstrated loss of accommodative function underwent Laser anterior ciliary excision in both eyes. Inclusion criteria comprised astigmatism less than 1 diopter (D) in the manifest refraction in both eyes, and distance-corrected visual acuity (DCVA) equal to or better than 20/40 in both eyes. Less than 0.50 D difference existed between manifest and cycloplegic refractions.

Data is reported on all patients through 12 months postoperatively $(\mathrm{N}=26)$. There were no statistically significant changes in uncorrected-distance visual acuity (UCVA) or spherical equivalent $(p=0.283 ; S D=0.10)$. The intermediate $(60 \mathrm{~cm})$ and the near $(40 \mathrm{~cm})$ visual acuities showed statistically significant improvement. Postoperatively, uncorrected-intermediate visual acuity (UIVA) was 20/32 or better in 95\% of subjects; $20 / 25$ in $85 \%$, and $20 / 20$ in $70 \%(p=0.042 ; S D=0.14)$. Uncorrected-near visual acuity (UNVA) was 20/32 in $85 \%$ of subjects, $20 / 25$ in $75 \%$, and $20 / 20$ in $25 \%(p=0.0007 ; S D=0.14)$. Distance-corrected intermediate visual acuity (DCIVA) was $20 / 3295 \%$ of subjects, $20 / 25$ in $90 \%$, and $20 / 20$ in $75 \%$ ( $p=0.001 ; S D=0.09)$. Distance-corrected near visual acuity (DCNVA) was $20 / 32$ in $85 \%$ of subjects, $20 / 25$ in $80 \%$, and $20 / 20$ in $25 \%(p=0.0008 ; S D=0.11$ ) (Figure 2) (ASCRS, Boston, MA, 2014).

Laser anterior ciliary excision appears to be a safe procedure for the restoration of accommodation in aging adults. Changing the biomechanical properties in the sclera appears in this IRB monitored pilot study to improve dynamic accommodative range for both intermediate and near vision. Further investigation must be performed to further assess efficacy and long-term stability.

\section{INTRODUCTION}

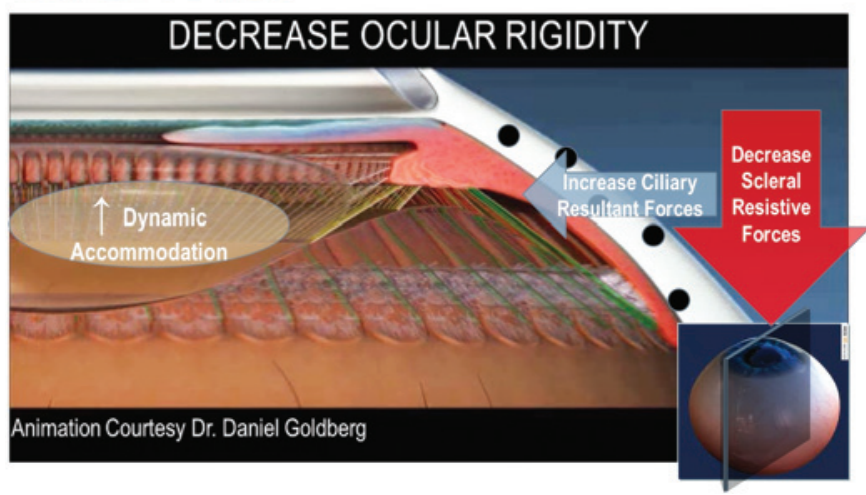

Figure 1. Caption "Model for accommodation and theory of reciprocal zonular action. Courtesy of Daniel Goldberg, MD".

\section{UCVA Summary Data @ 12 mos post op}

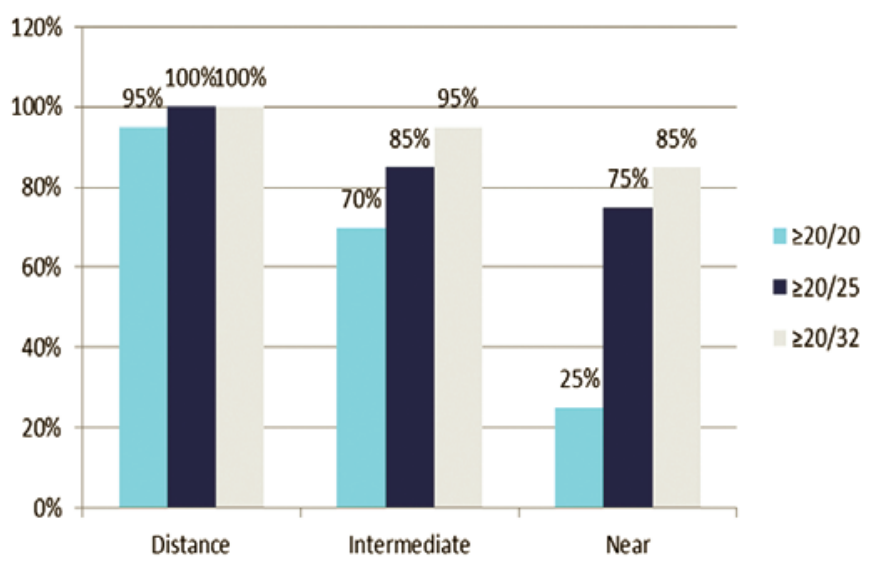

DCVA Summary Data @ 12 mos post op

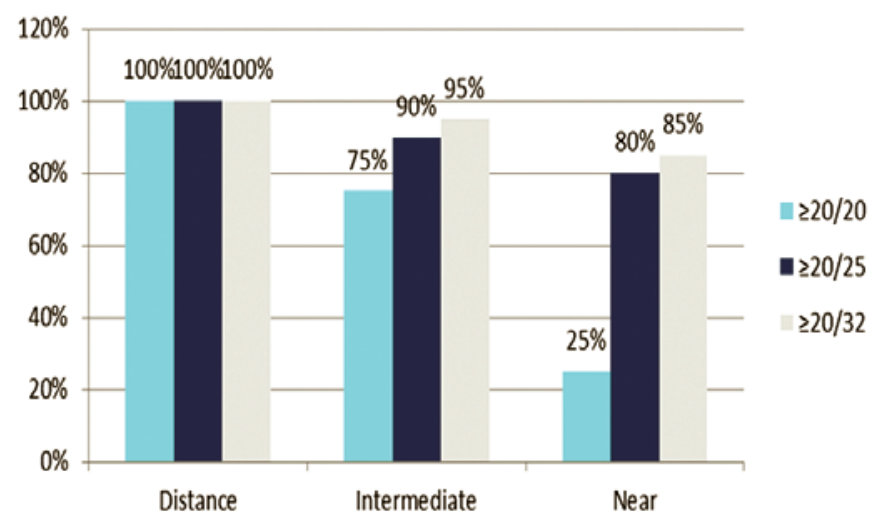

Figure 2. Caption "Visual outcomes 12 months after Laser Anterior Ciliary Excision". 


\section{REFERENCES}

1. Goldberg DB. Computer-animated model of accommodation and theory of reciprocal zonular action. Clin Ophthalmol. 2011;5:1599-66.

2. Croft MA, Nork TM, McDonald JP, Katz A, Lutjen-Drecoll E, Kaufman PL. Accommodative movements of the vitreous membrane, choroid and sclera in young and presbyopic human and nonhuman primate eyes. Invest Ophthalmolo Vis Sci. 2013 54(7):5049-58.

3. Lütjen-Drecoll E, Kaufman PL, Wasielewski R, Ting-Li L, Croft MA. Morphology and accommodative function of the vitreous zonule in human and monkey eyes. Invest Ophthalmol Vis Sci. 2010;51(3):1554-64

4. Pallikaris IG, Kymionis GD, Ginis HS, Kounis GA, Tsilimbaris MK. Ocular rigidity in living human eyes. Invest Ophthalmol Vis Sci. 2005;46(2):409-14.

5. Pallikaris IG, Kymionis GD, Ginis HS, Kounis GA, Christodoulakis E, Tsilimbaris MK Ocular rigidity in patients with age-related macular degeneration. Am J Ophthalmol.
2006;141(4):611-5. Comment in: Am J Ophthalmol. 2006;142(4):706-7; author reply 707: Am J Ophthalmol. 2006;141(4):731-2

6. Dastiridou Al, Tsironi EE, Tsilimbaris MK, Ginis H, Karyotakis N, Cholevas P, et al. Ocular rigidity, outflow facility, ocular pulse amplitude, and pulsatile ocular blood flow in open-angle glaucoma: a manometric study. Invest Ophthalmol Vis Sci. 2013;54(7):4571-7.

7. Wilde GS. Measurement of human lens stiffness for modelling presbyopia. Treatments [Thesis]. Oxford: Brasenose College, University of Oxford; 2011.

8. Hipsley A, Waring GO, Wang J, Hsiao E. A novel method using collagen cross-linking to evaluate the ability of the LaserACE procedure to decrease ocular rigidity as it relates to the efficiency of intra ocular accommodative forces. Poster session presented at: Annual Meeting of the American Society of Cataract and Refractive Surgeons, 2013 Apr 19-23; San Francisco, CA.

9. Hipsley A. Compelling Findings for restoring natural dynamic accommodation using dynamic abberometry. Poster presented at: Annual Meeting of the American Society of Cataract and Refractive Surgeons 2011 Mar 25-29; San Diego, CA.

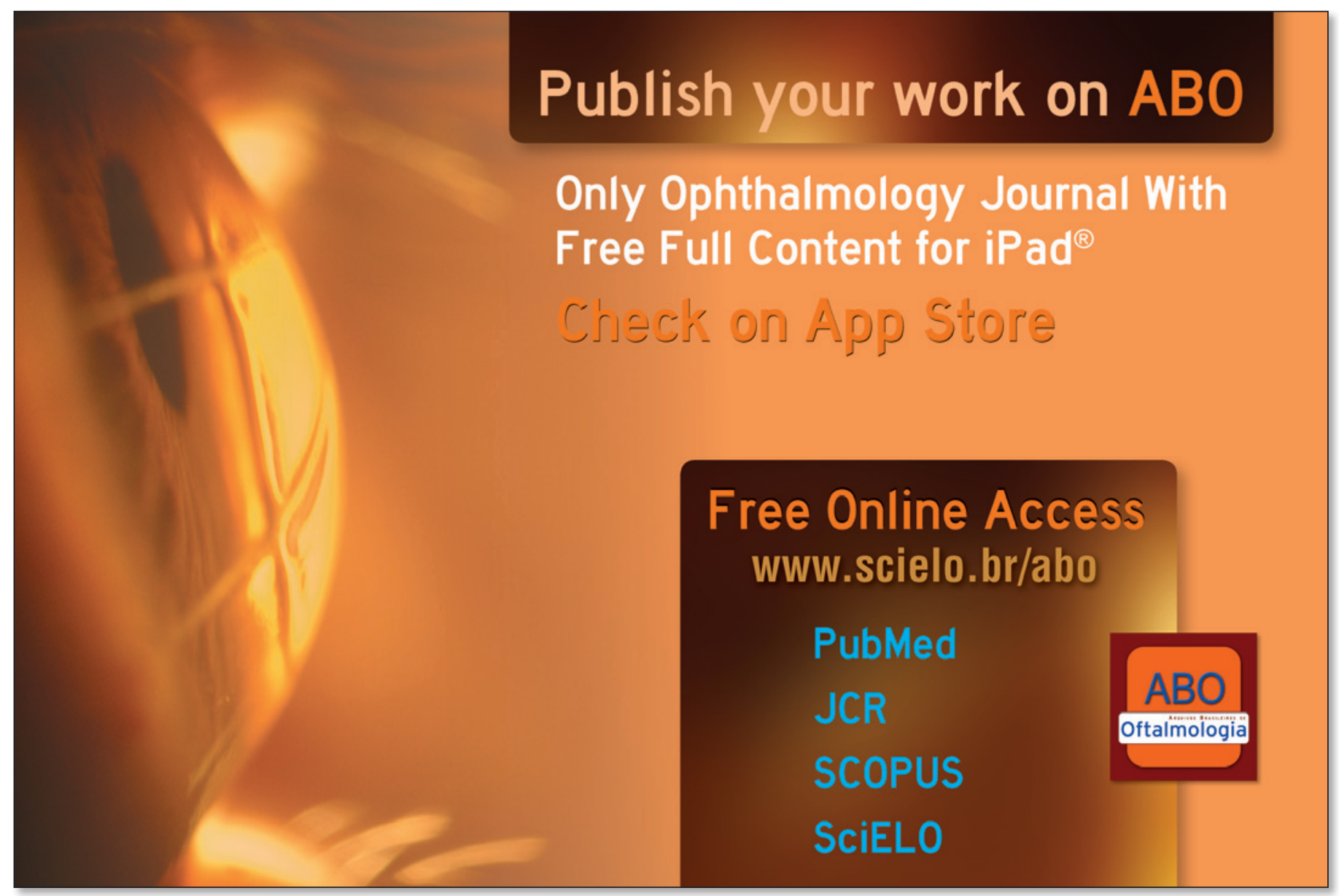

